\title{
A Knowledge-Extraction Experience in Anticoagulation for Early Postoperative Cardiac Valvular Surgery
}

\author{
MA Simonet ${ }^{1}$, A Boignard ${ }^{2}, \mathrm{~V} \mathrm{Bach}^{2}, \mathrm{~S}_{\text {Tramaille }}^{2}$, \\ M Simonet ${ }^{1}$, D Blin ${ }^{2}$ \\ ${ }^{1}$ Université Joseph Fourier, Grenoble, France \\ ${ }^{2} \mathrm{CHUG}$, University Hospital, Grenoble, France
}

\begin{abstract}
Cardiac valvular prosthesis surgery requires an anticoagulant treatment whose management is particularly difficult in early postoperative surgery. The capacity to avoid both thromboembolic and haemorrhagic risks relies on the integration of numerous parameters. In this paper, we present an experience of Knowledge Extraction from clinical and biological data from a cardiac surgery department of the University Hospital of Grenoble. The purpose of our study is to analyse the different types of decision, to detect possible anomalies in decisions, and to try to provide some computer-assisted help for these decisions. Using the Decision Tree approach provided final decisions that are consistent with clinical results and made explicit some implicit parameters used in the prescription task. This study also provided a better understanding of the behaviour of the prescribers, with some unexpected results.
\end{abstract}

\section{Introduction}

Knowledge Extraction (KE) is a way to produce a Decision Support System (DSS) in the medical field when expert knowledge is missing or cannot easily be made explicit, and when data on medical practice is available. This was the situation at the Grenoble University Hospital CHUG (Centre HospitaloUniversitaire de Grenoble) in the cardiac surgery department, concerning the problem of anticoagulant prescription in early postoperative cardiac valvular surgery. During the 7 to 10 days hospitalization period following the pose of a cardiac valve, the risks of thrombosis and of hemorrhage are particularly high, which makes necessary repeated controls of coagulation parameters.

The European project Noesis [1] offered the opportunity to start a study on Knowledge extraction from this data in order to feed the Decision Support Framework which was designed in the course of this project [2]. The DSF methodology to produce a Decision Support System (DSS) for a specific problem relies on a Decision Tree (DT) that is further transformed into fuzzy rules in order to take into account non-strict frontiers between discrete values. This methodology is currently being studied on various domains, among which anticoagulant prescription, based on data from digitalized patient files.

In this paper we present the Knowledge Extraction process and its result. As the Noesis DSS has not yet been tested, we limit our study to the DT that was first built from clinical data. Surprisingly, the main interest of this study appeared to be the clinical analysis of the data exhibited by the DT process. Before presenting this clinical analysis we recall the anticoagulant prescription problem in early postoperative cardiac valve surgery, we present the data collection process and we recall the DT principles.

The analysis of the DT and of associated data showed unexpected doctors' behaviour in some situations but mainly made explicit some parameters they implicitly take into account in their daily anticoagulant prescription task. These results will help designing a computer-system to support this task.

There is no international consensus on the drugs to use, their administration mode and the required dose [3][4]. In France most anticoagulation protocols use heparin and/or calcic heparin before the use of warfarin (antivitamin K). Monitoring is based mainly on ACT (Actived Cephalin Time) and INR (International Normalized Ratio). For every patient a decision on the dose of anticoagulant is made by a physician, one to three times a day, according to several parameters.

Some parameters are linked to the patient (age, atrial fibrillation, size of left atrium, previous thrombo-embolic accidents, creatinine level), to the type of prosthesis (biological or mechanical) and the localisation of the prosthesis (mitral or aortic), to the level of coagulation control, to the variations of controls (rapid decrease or rapid increase) and to the dose of anticoagulation drug (with respect to the patient's weight). Mechanical 
prostheses and mitral position have a higher risk of thromboembolism and require a higher level of anticoagulation.

Physicians are of various age and experience and are not all experts in anticoagulant prescription, all the more so that they need to take into account a number of parameters too large to be easily integrated.

The purpose of our study is to analyse the different types of decision, to detect possible anomalies in decisions, and to try to provide some computer-assisted help for these decisions.

\section{Methods}

\subsection{Population}

182 patients (between January 2004 and June 2006) suffering of isolated valvulopathy were selected. During their visit in the cardiac surgery department, patients have between two and three prescriptions of anticoagulant a day, providing 2487 anticoagulant prescription decisions.

Given the complexity of analysing mixed prescriptions, we decided to start our study by considering prescriptions involving only heparin alone, which give 570 decisions.

\subsection{Decision protocol and data collection}

A protocol has been designed in the cardiac surgery department in order to guide the doctors' prescriptions. In this protocol, patients are distributed into three groups according to their risk level. A score is calculated, ranging from 30 to 56, by considering the following parameters: localisation of valve (aortic or mitral), type of the valve (mechanical, biological, valvuloplasty) and Auricular Fibrillation (AF). The score is higher when a risk factor is present (e.g., mitral mechanic valve, recent AF). The result is presented as a three-valued risk level. Table 1 shows the ACT limits according to the risk level.

Table 1. ACT limits according to thrombosis and hemorrhage risk

\begin{tabular}{|l|c|c|c|}
\hline Risk & Score value & $\begin{array}{c}\text { ACT } \\
\text { lower limit }\end{array}$ & $\begin{array}{c}\text { ACT } \\
\text { upper limit }\end{array}$ \\
\hline Moderate & 30 & 50 & 80 \\
\hline High & $31 \leq \ldots \leq 40$ & 60 & 80 \\
\hline Very high & $\geq 41$ & 65 & 90 \\
\hline
\end{tabular}

The standard decision consists in increasing the dose when the ACT value is too low (thrombosis risk), decreasing the dose when the ACT value is too high (hemorrhagic risk) and keeping it equal when the ACT value is between the protocol limits.

Data was collected from paper files (anticoagulant type and dose, heart rate), from a biological database (ACT, creatinine, hemoglobin, ...) and merged with data collected during the doctors' visits (AF). The resulting database was designed to support statistical analysis. Each actual decision was controlled by another doctor (expert) and his/her conclusion (expert decision) was also recorded in the database.

\subsection{Decision tree methodology}

Decisions Trees are a supervised learning method, which can be easily explained to the doctors, because it follows a logical pattern of thought. A decision Tree begins by a root node, which is the best variable to take a decision. This root node is split into two child nodes, which are variables to help taking the decision. This process is done recursively until arriving to a final node (leaf) that represents the final decision.

The building of the tree starts at the root node, which includes all the patients in the learning dataset. Starting with this node, the DT building software finds the best possible variable in order to split the node into two child nodes. The process is stopped when either (1) there is only one observation in each of the child nodes, (2) all the observations in the child node have an identical distribution of predictor variables, making further splitting impossible, or (3) an external limit on the number of levels in the maximal tree has been set by the user [5]. We used the GINI index to build the trees and the cross validation method to validate our Decisions Trees.

\subsection{Variables}

The target variable is the final decision to increase the dose, decrease the dose or keep it equal. The expert's decision was used as the target variable to build the Decision Tree.

Biological variables (e.g., hematocrit, hemoglobin) are rarely used by doctors. Moreover, they have many missing values (up to $70 \%$ ), which is problematic for data analysis. Consequently, they were not used in the study.

In order to exhibit the doctors' practices by mining the data, it is important to select as variables the parameters they actually use to build their decision. Several sets of variables were tested. The best results were obtained with the following set.

- ACT_In_Limits synthesises the patient's anticoagulation state according to the risk level and the ACT value

- BMI (Body Mass Index): the dose prescribed to the patient is much dependent on his/her size and weight.

- VarACT: ACT variation compared with the last ACT value

○ Days_After_Surgery: the risk decreases with time. It is coded as an integer value 
Table 2. Coding of discrete values.

\begin{tabular}{|c|c|c|}
\hline Variable & Limits & Code \\
\hline \multirow{3}{*}{ ACT_In_Limits } & $<$ lower limit & ACT_Low \\
\hline & $\begin{array}{l}\text { lower limit } \leq \ldots \\
\leq \text { upper limit }\end{array}$ & ACT_Good \\
\hline & $>$ upper limit & ACT High \\
\hline \multirow{4}{*}{ BMI } & $<18,5$ & Thin \\
\hline & $18,5 \leq \ldots<25$ & Normal \\
\hline & $25 \mathrm{c} \ldots<30$ & Overweight \\
\hline & $\geq 30 \%$ & Obesity \\
\hline \multirow{3}{*}{ VarACT } & $<-30 \%$ & High decrease \\
\hline & $>30 \%$ & High increase \\
\hline & $-30 \% \leq \ldots \leq 30 \%$ & ACT_stable \\
\hline
\end{tabular}

\section{Results}

Several Decision Trees have been built. They were evaluated according to their relative cost. The tree with the lowest relative cost (i.e., the "best" tree) we obtained is presented in Fig 1. Each leaf in the tree corresponds to a final decision (by the expert). The most probable variable (in bold) expresses the decision most frequently taken in this situation. It also represents the final action if the DT were used as a decision support system.

The most discriminating variable is ACT_In_Limits, which corresponds to actual clinical practice. The value of the relative cost $(0.250+/-0.024)$ indicates that the conclusion proposed in each leaf node is consistent with the data set from which the DT has been built, hence with the medical expert decisions ( $75 \%$ are well-classified).

\section{Discussion and conclusions}

The DT in Fig. 1 shows that the ACT variation (Var_ACT) and the corpulence of the person (BMI) are important parameters in the medical decision. This was implicitly known to influence the decision, but the DT made it explicit.

Fig. 1 shows that in situations where the ACT is too high, while the standard decision would be to decrease the dose, this decision is taken in only $80 \%$ of all cases, while in $20 \%$ cases an equivalent dose is prescribed. In situations where the ACT is too low, the standard decision to increase the dose is taken in $97 \%$ of all cases. It shows that doctors, consciously or not, are more afraid of the trombosis risk due to a too low anticoagulation than of the hemoragge risk due to a too high anticoagulation. It is confimed in situations where despite ACT being within the protocol limits we observe in $34 \%$ of all cases an increase in doses and a decrease in only $2 \%$.

In order to evaluate the consequences of the medical decision we have studied the next ACT value in situations where the doctors took the standard decision (according to the protocol) or not (Tables 3a, 3b). We have emphasised (in bold) the lines corresponding to the desired result, i.e., when the ACT is within the protocol limits.

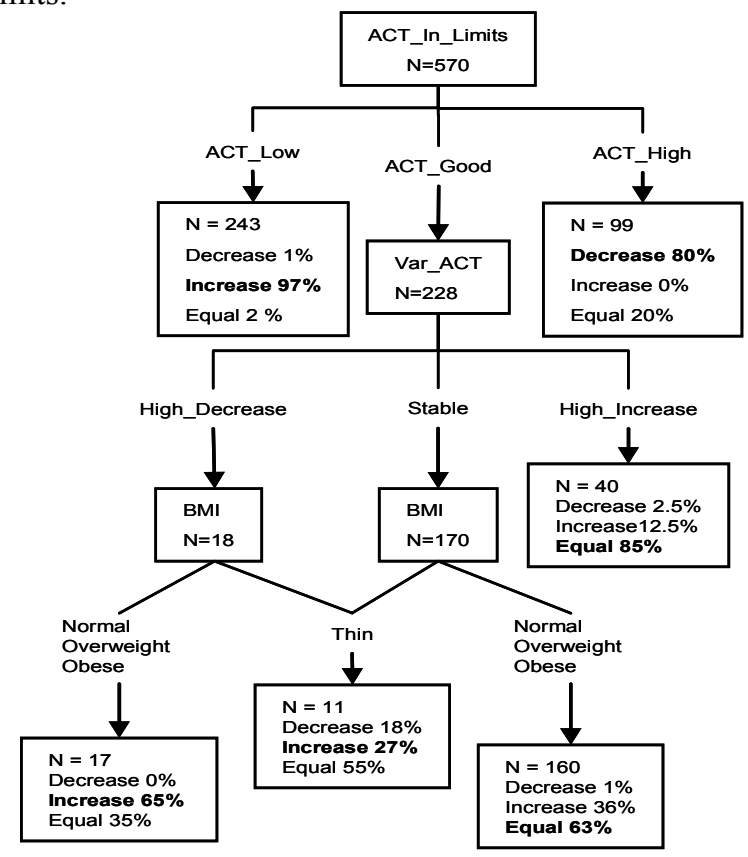

Figure 1. Decision Tree for heparin decisions.

Tables $3 \mathrm{a}$ and $3 \mathrm{~b}$ show the situations where the prescriber and the expert agreed on the decision, which we call the "ideal decision". Table 3a describes the situation where the ideal decision is the standard decision, and table $3 \mathrm{~b}$ describes the situation where the ideal decision is not standard. The number of decisions is put within square brackets (e.g., [13]).

The first row in Table 3a shows that when the current ACT is too low, despite a "good" decision, i.e., following the protocol, the next ACT value is within the desired limits only in $31 \%$ of the cases. The ACT value remains too low in $56 \%$ of the cases, which may be due to an insufficient dose increase; it becomes too high in $13 \%$ of the cases, which may be due to a too high dose increase. The same reasoning applies to each row in table $3 \mathrm{a}$. Therefore, despite appropriate decisions, the expected result is obtained only in $41 \%$ of the cases. This demonstrates the interest of checking frequently the coagulation level and adjusting the dose accordingly.

The global conclusion of Table $3 b$ is that when disobeying the protocol, the rate of good results is higher $(65 \%)$ than when following it in a strict manner

This seems to indicate that doctors take into account parameters that are not considered in the protocol, which reinforces the conclusions concerning the implicit parameters.

This work shows the difficulty of anticoagulant prescription in early postoperative cardiac surgery. The 
ACT of patients for whom the standard decision has been taken can go beyond the limits, whereas a non-standard decision can lead to a new ACT within limits. It highlights the importance of controlling many times a day each ACT in order to adjust the dose frequently.

Table 3a. Effect of standard decision on the next ACT when the prescriber and the expert agree.

\begin{tabular}{|c|c|c|}
\hline $\begin{array}{l}\text { Current } \\
\text { ACT value }\end{array}$ & $\begin{array}{c}\text { Actual } \\
\text { Decision } \\
\text { (standard) }\end{array}$ & Next $A C T$ value \\
\hline \multirow{3}{*}{$\begin{array}{c}\text { ACT } \\
\text { Low [218] }\end{array}$} & \multirow{3}{*}{$\begin{array}{c}\text { Increase } \\
{[212] 97 \%}\end{array}$} & Within limits: [66] 31\% \\
\hline & & Under limit [119] 56\% \\
\hline & & Over limit [27] 13\% \\
\hline \multirow{3}{*}{$\begin{array}{c}\text { ACT } \\
\text { High [82] }\end{array}$} & \multirow{3}{*}{$\begin{array}{l}\text { Decrease } \\
{[64] 88 \%}\end{array}$} & Within limits: $[26] 41 \%$ \\
\hline & & Under limit [20] 31\% \\
\hline & & Over limit [18] 28\% \\
\hline \multirow{3}{*}{$\begin{array}{c}\text { ACT } \\
\text { Good [182] }\end{array}$} & \multirow{3}{*}{$\begin{array}{c}\text { Equal } \\
{[116] 63 \%}\end{array}$} & Within limits: [68] 59\% \\
\hline & & Under limit [35] 30\% \\
\hline & & Over limit [13] 11\% \\
\hline
\end{tabular}

Table 3b. Effect of non-standard decision on the next ACT when prescriber and expert agree.

\begin{tabular}{|c|c|c|}
\hline $\begin{array}{l}\text { Current } \\
\text { ACT value }\end{array}$ & $\begin{array}{c}\text { Actual } \\
\text { Decision } \\
\text { (Non- } \\
\text { Standard) }\end{array}$ & Next ACT value \\
\hline \multirow{3}{*}{$\begin{array}{c}\text { ACT } \\
\text { Low }[218]\end{array}$} & \multirow{2}{*}{$\begin{array}{l}\text { Equal } \\
{[4] 2 \%}\end{array}$} & Under limit [3] 75\% \\
\hline & & Within limits: [1] 25\% \\
\hline & $\begin{array}{c}\text { Decrease } \\
\text { [2] } 1 \%\end{array}$ & Under limit [2] 100\% \\
\hline \multirow{3}{*}{$\begin{array}{c}\text { ACT } \\
\text { High [82] }\end{array}$} & \multirow{3}{*}{$\begin{array}{c}\text { Equal } \\
{[18] 22 \%}\end{array}$} & Within limits: [9] 50\% \\
\hline & & Under limit [6] 33\% \\
\hline & & Over limit [3] 17\% \\
\hline \multirow{4}{*}{$\begin{array}{c}\text { ACT } \\
\text { Good [182] }\end{array}$} & \multirow{3}{*}{$\begin{array}{r}\text { Increase } \\
{[65] 36 \%}\end{array}$} & Within limits: $[45] 69 \%$ \\
\hline & & Under limit [6] 9\% \\
\hline & & Over limit [14] 22\% \\
\hline & $\begin{array}{l}\text { Decrease } \\
{[1] 1 \%}\end{array}$ & within limits [1] $100 \%$ \\
\hline
\end{tabular}

An important result of this study is a better understanding of the behavior of the prescribers: they prefer to ensure a high level of anticoagulation, with a resulting bleeding risk, than a low level, with a risk of thrombosis.

The conclusions produced by the Decision Tree show a good correspondence with expert decisions (75\%). The current DT could be used as a safeguard: if each actual decision was compared with the DT proposal. In case of conflict between the two decisions, the prescribers would be invited to reconsider their decision; this would ensure that decisions diverging from "normal" expert decisions are taken consciously.

However, this is not sufficient to implement a Decision Support System for daily practice. In order to design a computer-based system to support the prescription of anticoagulant in early postoperative cardiac valvular surgery, further studies must be carried out. Increasing the population of support data should refine the decision process and might exhibit other variables that were not statistically significant with the current data set of 570 decisions for heparin. Moreover, we are now conscious of the zones for which the decision process has to be more thoroughly studied.

Once a Decision Support System has been set up with the three possible conclusions: increase, decrease or equal dose, the following step will be to try quantifying the dose to prescribe.

\section{Acknowledgements}

This research was funded by the European Commission - (NOESIS project, IST-2002-507960).

\section{References}

[1] NOESIS project: http://www.noesis-eu.org

[2] Exarchos TP, Tsipouras MG, Nanou D, Bazios C, Antoniou Y, Fotiadis DI. A Platform for wide-scale integration and visual representation of medical intelligence in cardiology: the decision support framework. Computers in Cardiology 2005; 32: 167-170.

[3] Kulik A, Rubens FD, Baird D, Wells PS, Kearon C, Mesana TG, Lam BK. Early postoperative anticoagulation after mechanical valve replacement: a Canadian survey. J Heart Valve Dis 2006: 581-587.

[4] Kulik A, Rubens FD, Wells PS, Kearon C, Mesana TG, Van Berkom J, Lam BK. Early postoperative anticoagulation after mechanical valve replacement: a systematic review. Ann Thorac Surg 2006: 770-781.

[5] Breiman L, Friedman JH, Olshen RA, Stone CJ. Classification and regression trees. Wadsworth and Brooks Pacific Grove, California, 1984.

Address for correspondence

Maria-Ana Simonet

Institut Albert Bonniot - INSERM 823 - Equipe 8

BP 170 La Tronche

38042 Grenoble Cedex

FRANCE

Maria-Ana.Simonet@imag.fr 